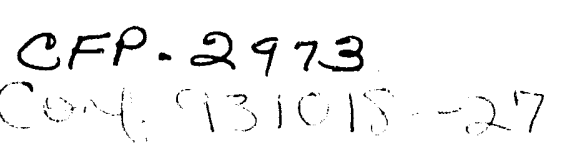

\title{
PLASMA VERTICAL STABILITY AND FEEDBACK CONTROL FOR TPX
}

\author{
C. E. Kessel, S. C. Jardin, G. H. Neilson \\ Princeton Plasma Physics Laboratory \\ P. O. Box 451 \\ Princeton, New Jersey 08543
}

\begin{abstract}
The $\mathrm{n}=0$ axisymmetric vertical stability and vertical position control have been examined for the Tokamak Physics Experiment. The passive stabilization is accomplished by using stabilizer plates close to the plasma. The present configuration is found to provide robust stability over a wide range of plasma parameters. The active feedback control of the plasma vertical position is done using coils located inside the vacuum vessel. These are required to control random disturbances leading to $\leq 1.0 \mathrm{~cm}$ RMS displacements from the midplane, and acceptable coil currents and voltages are found.
\end{abstract}

\section{INTRODUCTION}

Analysis was done for the Tokamak Physics Experiment (TPX) to determine the passive stabilization and feedback control requirements for the plasma vertical position. The $\mathrm{n}=0$ axisymmetric vertical stability is examined using the TEQ equilibrium and stability code[1, 2], and vertical position control is examined with the Tokamak Simulation Code[3] (TSC). The major plasma parameters for TPX are $\mathrm{I}_{\mathrm{p}}=2.0 \mathrm{MA}, \mathrm{R}=2.25 \mathrm{~m}, \mathrm{a}=0.50$ $\mathrm{m}, \kappa=2.0, \delta=0.8$ and $\mathrm{B}_{T}=4.0 \mathrm{~T}$. The plasma, structure, and poloidal field coil model used in the analysis is shown in Fig. 1.

A vertically elongated plasma is unstable to motion in the vertical direction and will move on an Alfven time scale (microseconds). In order to slow the plasma motion down to allow some form of feedback control, conducting structure must be located close to the plasma. In the presence of the structure the plasma is still unstable, but now moves on the time scale of the eddy currents in the structure (milliseconds). Acrive feedback control coils, that can respond in milliseconds and provide a radial magnetic field, can then be used to maintain the plasma vertical position indefinitely.

\section{PLASMA VERTICAL STABILITY}

The plasma vertical instability will be characterized by a linear growth time $y_{y}=1 / \gamma_{g}$ ) for the unstable plasma vertical motion, in the absense of active feedback control.

$$
Z(t)=Z_{o} e^{\gamma_{g} t} .
$$

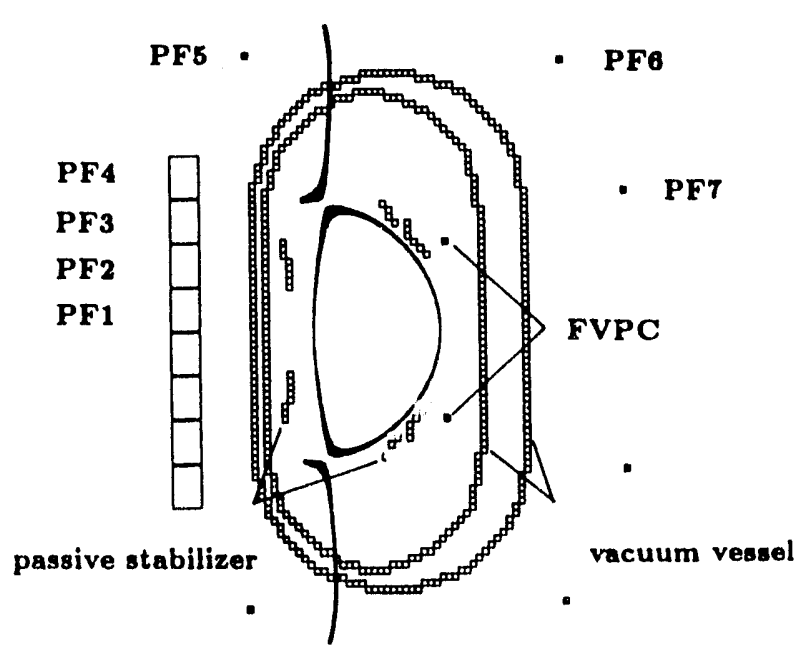

Figure 1: Model of plasma, structure, and coils used in analysis.

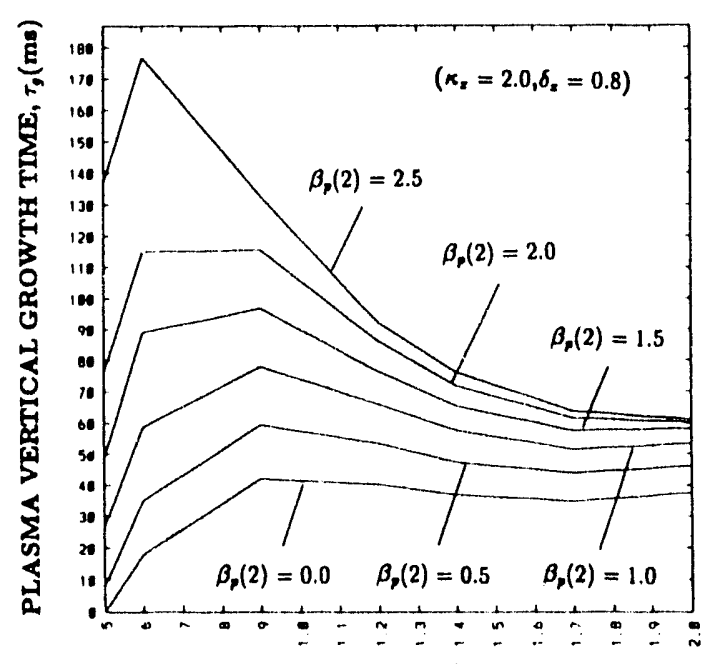

Plasma internal inductance, $l_{i}(3)$

Figure 2: Plasma vertical growth time as a function of the current distribution and pressure. 
The factors that determine the vertical growth time are the geometry and electrical resistance of the surrounding conducting structure, and the plasma current distribution, pressure, and elongation.

When the plasma moves vertically it induces eddy currents in the surrounding conducting structure. Whether these eddy currents are sufficient to retard the plasma motion will depend on how close the structure is to the plasma and its poloidal coverage. This retarding effect is characterized by a stability factor $\left(f_{s}\right)$ and represents a purely inductive response of the structure.

$$
f_{s}=1+\frac{\tau_{g}}{\tau_{L / R}} .
$$

Here $\tau_{L / R}$ is the time scale of the currents induced in the structure. When $f_{0}=1$ the structure is ineffective in retarding the plasma motion and it moves on the Alfven time scale. Once the structure's geometry provides $f_{3}>1$, the degree to which the surrounding structure slows the plasma depends on the structure resistance, in particular, the structure resistivity, thickness, and toroidal extent. The plasma vertical growth time $\left(\tau_{g}\right)$ is then a measure of this. This growth time is proportional to the structure eddy current time scale $\left(\tau_{L / R}\right)$.

The requirement for the surrounding conducting structure in TPX is to provide for a stability factor $f_{s} \geq 1.2$ for all anticipated plasmas. The vacuum vessel is too far from the plasma to provide for this requirement, so that passive stabilizer plates are introduced close to the plasma. The position of these plates is excluded from the outboard midplane (ports), the divertor entrances (top and bottom), and the plasma scrape-off regions (inboard and outboard). The plates must be made of a material with sufficient conductivity to make $\tau_{g}$ long, so that a reasonable feedback control system can be designed.

There is a wide range of plasmas that are required in TPX to achieve the mission of the device. For vertical stability, the plasma is completely described by its current distribution $\left(l_{i}\right)$, pressure $\left(\beta_{p}\right)$, and elongation $(\kappa)$. At full plasma current $(2.0 \mathrm{MA})$ the range is given by,

$$
\begin{gathered}
0.5 \leq l_{i}(3) \leq 1.2 \\
0.0 \leq \beta_{p}(2) \leq 2.5 \quad 0.0 \leq \beta_{N} \leq 5.0 \\
\kappa_{x}=\kappa_{x}^{\max }\left(l_{i}, \beta_{p}\right) .
\end{gathered}
$$

Here $\kappa_{x}$ is the plasma elongation at the separatrix surface, and $\kappa_{x}^{\max }$ refers to the maximum elongation allowed, for the given $l_{i}$ and $\beta_{p}$, that properly intercepts the divertor. The terms $l_{i}(3)$ and $\beta_{p}(2)$ refer to the definitions in rof[4]. At lower plasma current $(\sim 1.0 \mathrm{MA})$ the range is given by,

$$
\begin{gathered}
0.5 \leq l_{i}(3) \leq 1.5 \\
0.0 \leq \beta_{p}(2) \leq 5.0 \quad 0.0 \leq \beta_{N} \leq 7.0 \\
\kappa_{x}=\kappa_{x}^{\max }\left(l_{i}, \beta_{p}\right) .
\end{gathered}
$$

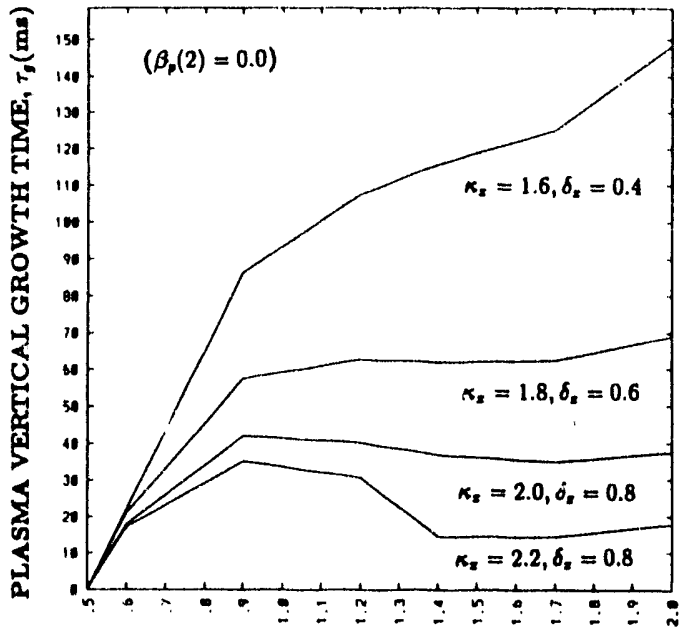

PLASMA INTERNAL INDUCTANCE, $l_{i}(3)$

Figure 3: Plasma vertical growth time as a function of current distribution for various elongations, at $\beta_{p}=0.0$.

The resulting passive stabilizer geometry, which allows as much of the required plasma operating space as possible and keeps the structure within the allowed envelope, is shown in Fig. 1. There is an inboard and outboard pair of up-down symmetric plates. The plates are connected into saddle configurations with a single toroidal break to decouple them from the ohmic circuit. The plates are made of GLIDCOP, are $0.025 \mathrm{~m}$ thick, and operate at $150^{\circ} \mathrm{C}$, corresponding to an electrical resistivity of twice that of pure copper at $20^{\circ} \mathrm{C}$. The plates are not electrically insulated from the vacuum vessel. The vertical stability and control analyses are axisymmetric (2-D), and since the saddle plates are 3-D structures, an axisymmetric equivalent model is generated by the electromagnetics code SPARK[5], for use in these analyses.

For the defined passive stabilizer, the eddy current time constant $\tau_{L / R}$ is $130 \mathrm{~ms}$, leading to a minimum plasma vertical growth time of $26 \mathrm{~ms}$. The plasmas that have vertical growth times less than this value are excluded from the operating space, and must be avoided during operation. These plasmas are those with very broad current profiles at low pressure. Figure 2 shows the plasma vertical growth time as a function of $l_{i}(3)$ and $\beta_{p}(2)$. The plasmas that have growth times less than the minimum (2f $\mathrm{ms}$ ) are evident. The plasmas with the most peaked and most broad current profiles have the worst stability. Figures 3 and 4 show the plasma vertical growth time as a function of $l_{i}(3)$ and $\kappa_{x}$ for $\beta_{p}(2)=0.0$ and 1.5. Lower elongations lead to longer growth times for most plasma current profiles, however at very broad current profiles there is no benefit. Increased pressure leads to more stable plasmas. In addition to these operating point plasmas, plasmas generated during startup and radially offset plasmas have been examined and show robust stability with the present passive stabilizer configuration. 


\section{VERTICAL POSITION FEEDBACK CONTROL}

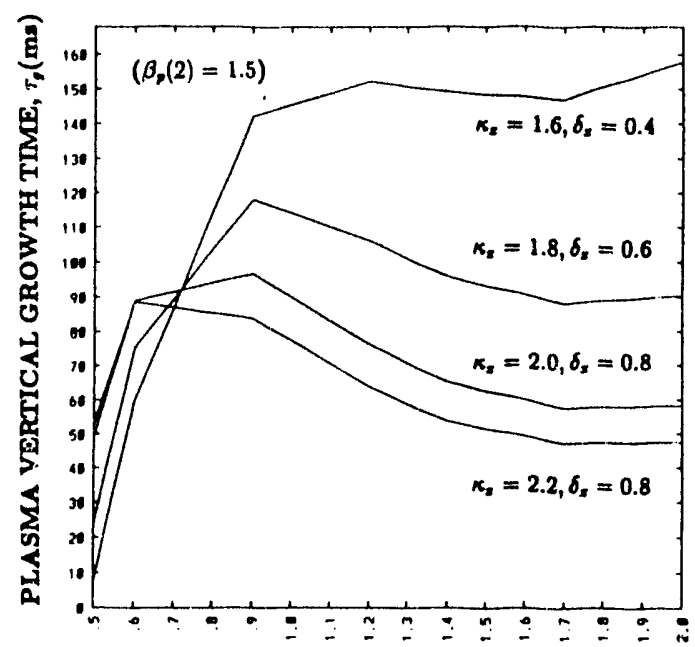

PLASMA INTERNAL INDUCTANCE, $l_{i}(3)$

Figure 4: Plasma vertical growth time as a function of current distribution for various elongations, at $\beta_{p}=1.5$.

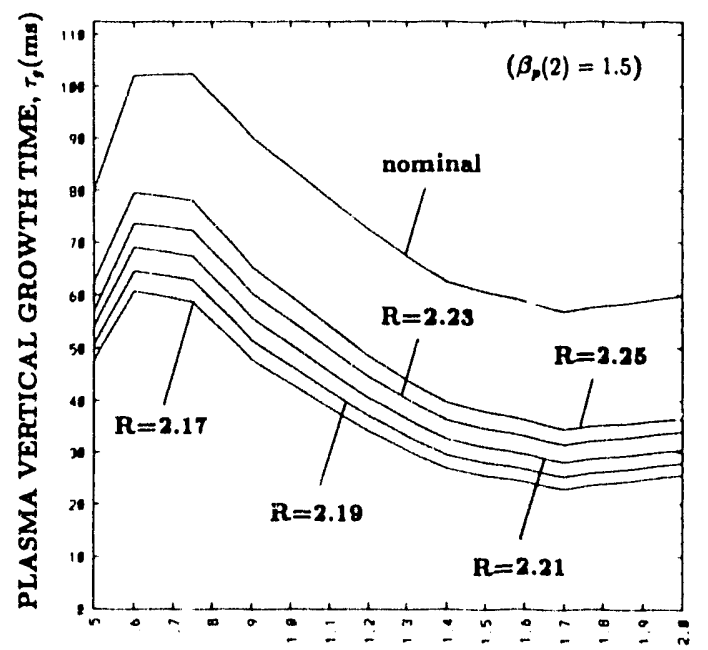

PLASMa INTERNal INDUCTANCE, $l_{i}(3)$

Figure 5: Plasma vertical growth time as a function of current distribution for various major radii, at $a=0.50$ and $\beta_{p}=1.5$.

For example, Fig. 5 shows the plasma vertical growth time as a function of $l_{i}(3)$ for various major radii with a reduced minor radius $(a=0.48)$. The nominal values are $R=2.25$ and $a=0.50$. This is important to assess the vertical stability of plasmas that experience ELMs or minor disruptions, leading to radial motion.
Once the surrounding conducting structure is sufficient to provide some retarding of the unstable plasma motion, a trade-off can be done between growth time $\left(\tau_{g}\right)$ and the feedback control power. The feedback control power (control coil currents and voltages) depend on the plasma vertical growth time, plasma current, reedback coil locations $\left(\mathrm{R}_{c}, \mathrm{Z}_{c}\right)$, and the plasma vertical displacement $\left(\Delta Z_{p}\right)$. In addition, the ability to control the plasma vertical position is strongly influenced by the power supply characteristics such as, maximum available voltage $\left(V_{\max }\right)$, voltage slew rate $(\mathrm{dV} / \mathrm{dt})$, time delays $\left(\tau_{d}\right)$, and available voltage at a given current. The goal is to control the plasma vertical position in spite of disturbances, and do so for all anticipated plasmas with a reasonable feedback control power. The requirement for TPX is that the feedback control system (control coils and power supply), for all anticipated plasmas within the defined surrounding conducting structure, provide for control of $1.0 \mathrm{~cm}$ RMS random displacements about the midplane. This requirement is motivated by the experimental vertical position trajectories observed on D-IIID, JET, and PBX-M. This is expected to represent the actual type of disturbance control encountered during steady state conditions of the discharge. The vertical position control is simulated in order to determine the feedback coil currents and voltages required. The worst case plasma is used ( $\left.\tau_{g}=26 \mathrm{~ms}\right)$, and the feedback control coils (referred to as the Fast Vertical Position Coils, FVPC) are located inside the vessel. It is assumed that the vertical position of the plasma is available for feedback. In order to determine the best gains a $2.0 \mathrm{~cm}$ step simulation is done. This provides a simple and direct way of assessing the plasma position response for various gain combinations.

The random disturbance is simulated by feeding the coils a random signal to move the plasma from position to position. The random signal is characterized by an amplitude $\left(S_{0}\right)$, an autocorrelation time $(\tau)$, and a period $(T)$. The random signal used is given by,

$$
S(t)=2 S_{o}\left[\frac{\pi \tau}{T}\right]^{\frac{1}{2}} \sum_{n=1}^{\infty} \exp \left(-\frac{\omega_{n}^{2} \tau^{2}}{8}\right) \cos \left(\omega_{n} t-\phi_{n}\right) .
$$

Here $\omega_{n}=2 \pi n / T$, and $\phi_{n}$ is a uniform random phase $\left(-\pi \leq \phi_{n} \leq \pi\right)$. The randorn disturbances are characterized by a $\Delta Z_{R M S}$ of $1.0 \mathrm{~cm}$ and a power spectral bandwidth of $\Delta \omega=1 / \tau_{g}$, where $\tau_{g}$ is the minimum plasma vertical growth time $(26.0 \mathrm{~ms})$. The autocorrelation time is related to the bandwidth by $\tau=\sqrt{\pi} / \Delta \omega$. In addition, a $1.0 \mathrm{~ms}$ time delay is included to represent measurement. processing and power supply activation. Figure 6 shows the time history of the plasma vertical position for a single random disturbance simulation. The required peak current and voltage are $35 \mathrm{k} \Lambda$-turns and $17 \mathrm{~V} /$ turn, respectively. The RMS current and voltage are $15 \mathrm{k} \Lambda$-turns and $12 \mathrm{~V} /$ turn, respectively. 


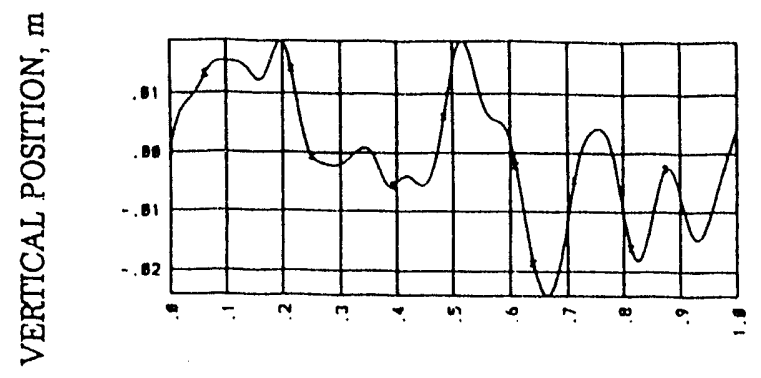

TIME, sec

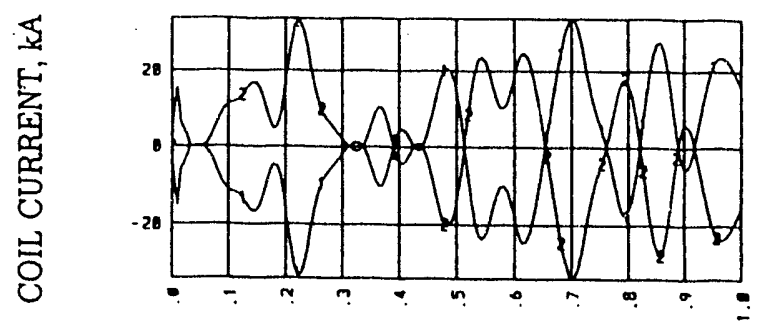

TIME, sec

Figure 6: The plasma vertical position and feedback coil current as function of time for the random disturbance control simulation

Analysis of the plasma vertical stability has led to the design of passive stabilizer plates that provide sufficient stabilizing influence for all plasmas in the wide range of plasma operating space $\left(l_{i}, \beta_{p}, \kappa_{x}\right)$ required for TPX. Dynarnic simulation of the feedback control system, with control coils located inside the vacuum vessel, has shown that the system can provide vertical position control for all anticipated plasmas with reasonable power requirements.

\section{References}

[1] L. D. Pearlstein and R. H. Bulmer, private communication.

[2] D. J. Strickler, N. Pomphrey, and S. C. Jardin, "Equilibrium Shape Control in CIT PF Design", Proc. 19th Symp. on Fusion Engr., Knoxville, p. 898, 1989.

[3] S. C. Jardin, N. Pomphrey, and J. Delucia, "Dynamic Modeling of Tranport and Position Control of Tokamaks", J. Comput. Phys., vol. 66, p. 481, 1986.

[1] N. A. Uckan and ITER Physics Group, "ITER Physics Design Guidelines", IAEA/ITER Report, ITER-TNPHI-05, 1990.

[5] D. Weissenburger, "SPARK Version 1.1: User's Manual", Princeton Plasma Physics Lab. Report, PPPL2494, 1988. 

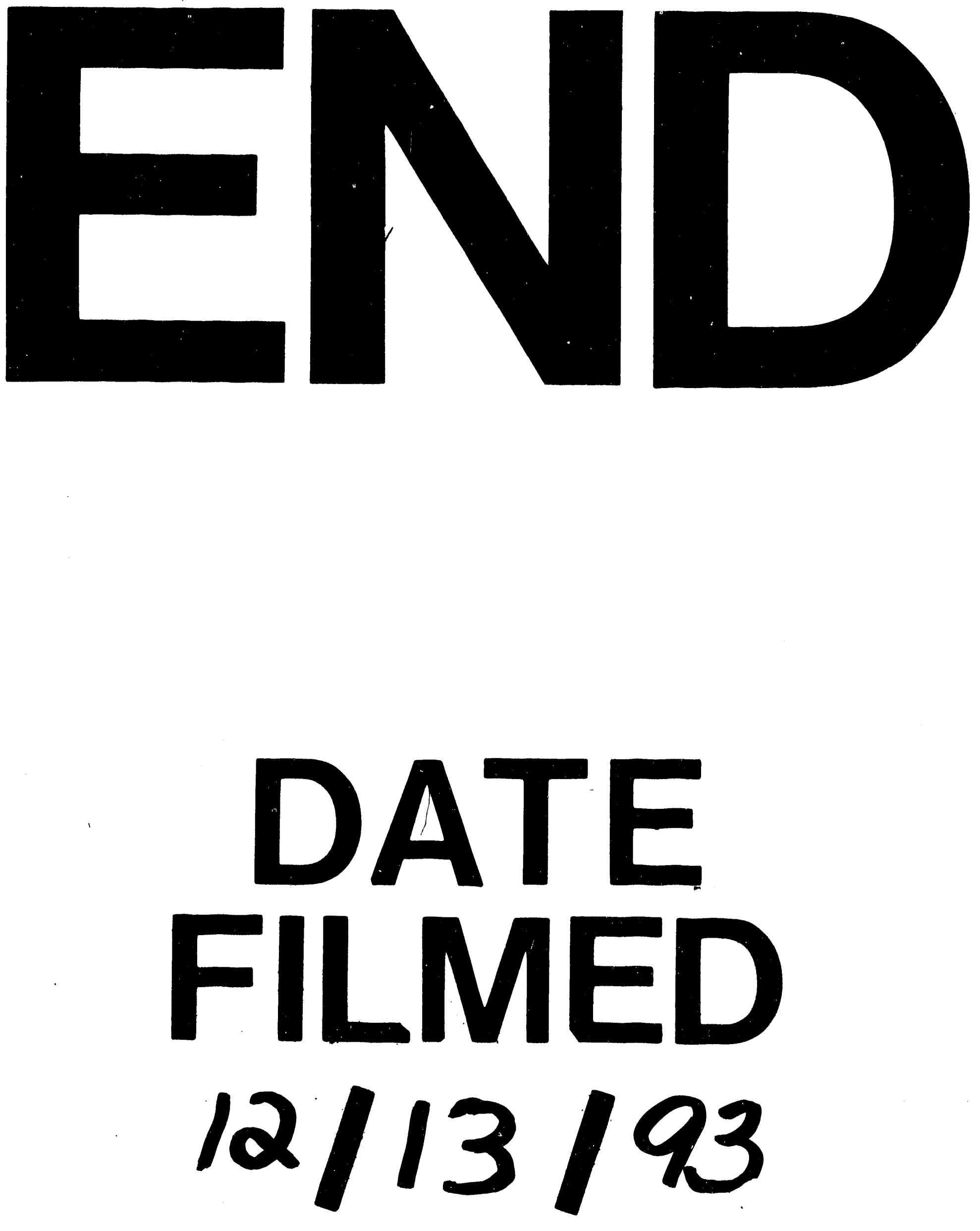
